\title{
This is my unit: CIVA at Sahlgrenska Hospital, Gothenburg, Sweden
}

Anna Cedergren, RN; CCN, Critical Care Nurse, central intensivvårdsavdelning (CIVA), Sahlgrenska Hospital, Gothenburg, Sweden.

E-mail: anna.y.cedergren@telia.com

Key words: critical care nursing intensive care unit $\quad$ Sweden

\section{SUMMARY \\ * This article gives an insight into our general intensive care unit, CIVA, one of Sweden's largest intensive care units. \\ * There are approximately 180 staff working in the unit. This includes nurses and auxiliary nurses. \\ * Nurses are involved in planning care, giving drugs, documenting progress and procedure, discussing care with the surgeons if necessary, and mentoring students. * Because of the wide variety of patients admitted, CIVA is a very stimulating unit to work in.}

\section{INTRODUCTION}

This article gives an insight into one of Sweden's largest intensive care units: central intensivvårdsavdelning (CIVA), which means general intensive care unit (ICU). The ICU is at the Sahlgrenska Hospital in Gothenburg. Together with two other hospitals in Gothenburg, Mölndal and Östra Hospital, they form Sahlgrenska University Hospital (SU). SU is closely linked with the University of Gothenburg, with a number of research projects going on.

$\mathrm{SU}$ is a public hospital serving a large area on the western coast around Gothenburg. With approximately 175,000 patients a year, it is one of the largest hospitals in Scandinavia, and has a wide variety of specialities such as cardiothoracics, transplantation, plastic surgery, paediatrics, neurosurgery and orthopaedics among others. Apart from CIVA, there are also a few other ICUs in the hospital: cardiac, neuro, infection and paediatric.

\section{PROFILE OF CIVA}

At CIVA, patients are admitted with respiratory failure, cardiovascular compromise, drug intoxication, cardiac arrest, liver/kidney transplant, septicaemia, hypothermia and other problems that require intensive care. It is also the main referral centre for trauma patients in the area. (Due to the number of injuries they can have, it is convenient to have these patients at Sahlgrenska Hospital, with access to a number of specialities). Sometimes CIVA will take neuro patients due to a shortage of beds in the neuro ICU. Children undergoing plastic surgery due to cranial deformations also go to this unit rather than the paediatric ICU because the plastic surgery department is in this building. Sometimes patients with liver failure, apart from being transplanted, are also given MARS treatment (a kind of liver dialysis). The unit also offers specialist medical care for patients who have overdosed on various drugs.

CIVA itself consists of two parts. The intensive care unit lies at one end and has ten beds (five rooms). Occasionally, one more room can be opened if we need to nurse an MRSA patient. At the other end, there is the post-operative unit which has 14 beds and is divided into three large rooms. There is also a unit downstairs with 20 beds for post-operative patients. In 2004, the ICU had approximately 1,700 patients and the post-operative unit approximately 3,400 patients.

Each side has a reception area. There are also a few offices dedicated to secretaries, the ward manager, a computer specialist, a clinical nurse specialist and physicians. Between these units there is a lunch room and a kitchen, an equipment room and a large drug cupboard/room. There are also two more rooms available for handing over, teaching or other activities.

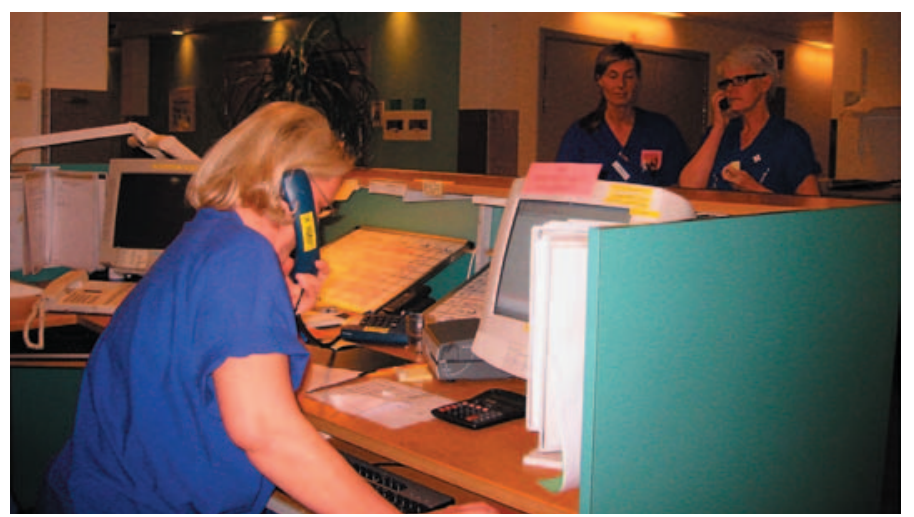

Photo 1: Organizing the bed situation

In the ICU, two patients are nursed in each room and between the rooms there is a sluice. Each room has its own drug cupboard and computer along with the usual ICU equipment such as monitors, ventilators, pumps and a couple of cupboards containing linen and hygiene and wound care utilities. Sometimes patients need treatments such as haemodiafiltration, liver dialysis (MARS), continuous electroencephalogram (EEG) monitoring or intracranial pressure monitoring. In these cases, additional equipment is brought into the room.

At the hospital, there is no high dependency unit where patients can stay before they go to the ward. However, sometimes more patients requiring intensive care are admitted than there are beds. This means that an ICU patient may need to lie on the post-operative side. Under these circumstances, they can swap places with a patient on ICU who is ready to go to the ward within a day or two. The average length of stay for ICU patients is approximately three 
days and for the post-operative patients it is usually less than 24 hours.

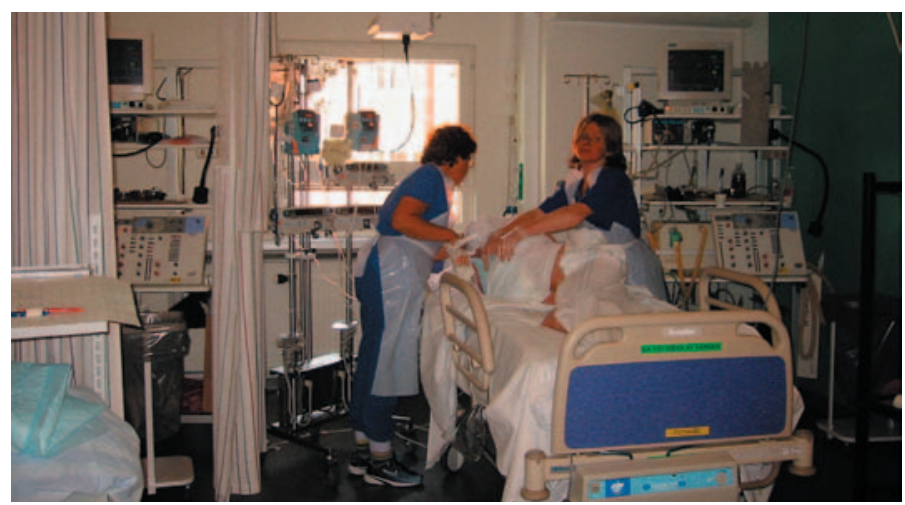

Photo 2: Bedside nursing

\section{Personnel}

This unit has a ward manager and an assistant ward manager. They have administrative duties and are not involved in the clinical work. There are also six team leaders. Apart from nursing patients, they are also responsible for allocating staff, overseeing the bed situation and carrying out annual performance reviews with the nurses and auxiliary nurses.

CIVA offers a variety of educational support. Each year, the unit organises two study days for all staff. Usually these take place outside the hospital and lecturers are invited to talk about topics that relate to intensive care. Recent topics include respiratory and cardiovascular failure and surgical methods. On these days, the staff actively participates in discussions. This spring, the topic was health and well-being and included an outdoor activity in the afternoon.

The hospital as a whole is working towards promoting health and well-being. Each year there is a cycling contest during the summer. Members of staff are encouraged to cycle to work and subsequently gain points for doing so. At the end of the summer there is a lottery and prizes can be won.

The unit also offers opportunities to take a variety of courses, some of which are organised by the hospital, such as trauma and advanced life support. The university offers courses for those who want to study towards a degree (not all nurses have a Bachelor of Science) or a Masters degree. These courses can be taken with a paid leave of absence.

At the nurses' meetings, which are held once a month, different people are invited to talk about various topics of interest. Recently, a transplant co-ordinator and staff from a clinical chemistry laboratory were invited to talk about their work. Visits are also made to other areas, such as the accident and emergency unit. At these meetings, other things are also discussed that are relevant to the day-to-day work.

\section{A TYPICAL DAY}

Day staff start work at 6:45am and the night nurse hands over untill 7:00am. The patients are looked over by nurses, auxiliary nurses and doctors in the morning. Usually some of the patients have been washed by the night staff. The auxiliary nurse washes the other patients and this is usually done by 10:00am. Depending on workload, the nurse will also lend a hand. If they are very busy, there is always a 'floater' who can help. The nurse looks at the patient's fluid balance, looks up blood results (and X-ray results) on the computer and plans for the ward round.

After the ward round, which is usually between 8:00am and 9:00am, there are a number of things that need to be done. Drugs need to be given, enteral feeding to be commenced, blood transfusions if necessary, physiotherapy including suctioning, wound care, etc. Patients that are well enough are mobilised in the morning (and also in the afternoon) with the physiotherapists.

In the middle of the day, there is usually more staff around. For about an hour, the morning and evening staff overlap. During this time, heavy patients such as trauma patients are mobilised, which requires up to six members of staff. Nurses and auxiliary nurses also spend a lot of time with relatives, both as support and to keep them up-to-date with what is happening with the patient.

\section{Nursing duties}

There are approximately 180 staff working in the unit. This includes nurses and auxiliary nurses, approximately half of which are qualified nurses. The nurses study for three years at university level and, to work in ICU, they then take a critical care course for a further year. This makes them ICU specialists. There is no grading system for registered nurses, such as there is in England for example. Auxiliary nurses study for two to three years and are delegated to do a number of nursing duties like suctioning, wound care and checking ventilators. They also do basic nursing care.

One nurse and one auxiliary nurse work together in an ICU room, nursing two patients. Usually, they stay with the same patients for the rest of the week. Nurses who have just started working in the unit are usually allocated to the post-operative unit. Here, two nurses and two auxiliary nurses work together in one room. Having one more nurse in the room gives a little more support initially. Also, new staff are not allocated to look after small children as this in itself is a big challenge.

All staff usually work in both areas. At times, one unit might be preferable over another for various reasons and this is taken into consideration when allocating staff. At present, not all nurses are trained to manage patients on haemodiafiltration. However, the aim is for all the nurses on the unit to undergo this training. Also, only a certain number of nurses are trained to use the MARS machine.

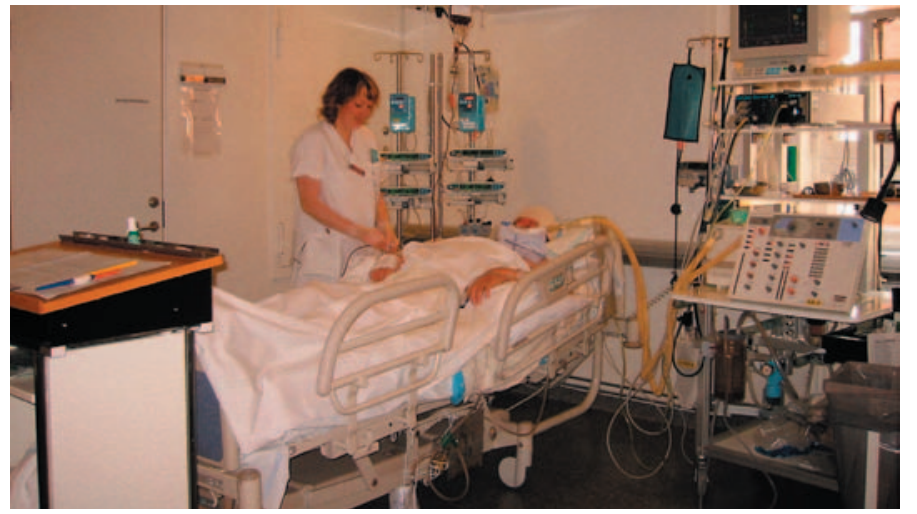

Photo 3: Administering blood to a transplant patient

Nurses plan the patients' care with other members of the multidisciplinary team. Usually they do the ward round together with a doctor and then they discuss what needs to be done with the patient. Sometimes the patient needs to be taken to the X-ray 
department either for an X-ray or computed tomography (CT) scan. Doctors do not normally accompany them on this transport unless the patient is particularly unstable.

Other aspects of the nurse's role are to give drugs, document progress and procedure, and discuss care with the surgeons if it is a surgical patient. Some tasks are delegated to the auxiliary nurses. Due to the large number of liver transplant patients in the unit, the nurses are invited to attend the transplantation board where these patients are discussed.

Nurses also take on students who are studying the critical care course. The unit regularly has a lot of students and they can stay for up to ten weeks. Typically, two nurses supervise one student for these weeks. During this clinical course, the students undertake a clinical examination with teachers from the university.

\section{Organising the rota}

The staff plan their own rota. Sometimes minor adjustments need to be made so that there is enough staff each day. Most staff work full-time, 38.25 hours a week, but there is also the possibility to work less. The working day is divided into three shifts: day (06:4515:00), evening (13:00-21:30) and night (21:00-07:00). There are some nurses who only work nights and some who only work days. A few of the nurses do both (80\% daytime and $20 \%$ nights). Recently, some changes have been made because of the shortage

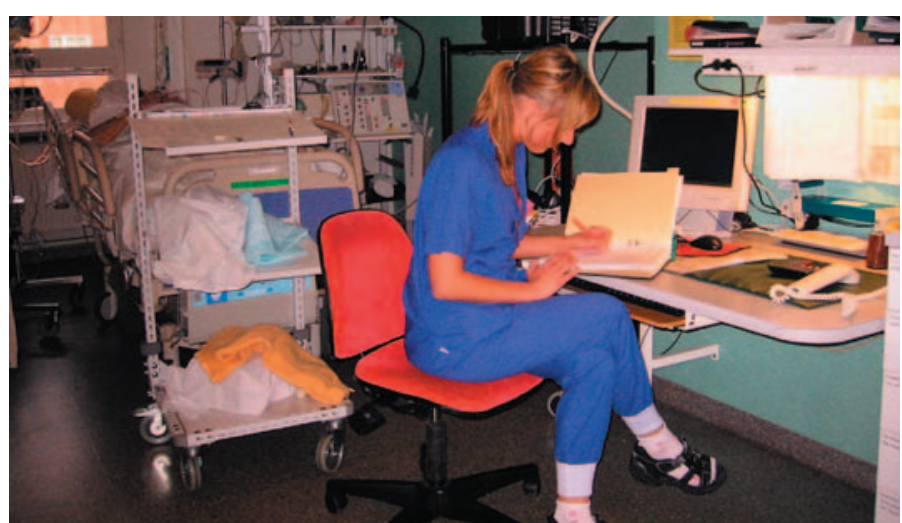

Photo 4: Counting the fluid balance of nurses on the night shifts. This means that new staff are only offered employment on a rotational basis, meaning they need to work $20 \%$ nights.

\section{PRACTICE DEVELOPMENT AND RESEARCH}

The unit has a number of interest groups, such as a respiratory group. They are currently looking at a standardised weaning programme for the unit. There are also groups who look at ethical issues, nutrition, wound care and pain relief. Members of these groups come to the nurses' meetings and give information. There is also a lot of information available on the unit's internet page.

Currently, there are three nurses (doctoral students at the university) doing research at CIVA. Their topic areas are 'End of life care', 'Memories of ICU and its effects on quality of life' and 'The importance of next of kin for the unconscious patients'. Recently, the unit has completed a study looking at patients' experiences of nursing care and environment, which was part of an international study.

At present, there is a group of physicians and nurses working on developing a medical emergency team/outreach team. They will offer advice to the wards regarding patients who could potentially need intensive care.

\section{SUMMARY}

Because of the wide variety of patients admitted, CIVA is a very stimulating unit to work in. Occasionally we treat patients with problems that we have not come across before and it is rare that we see patients with the same conditions in close succession. For these reasons, the unit has a large number of guidelines for various procedures and problems.

Initially, I think it could be a little overwhelming to work at CIVA because of its size and the fact that it has so many members of staff - it takes time to get to know everybody. However, the unit is an excellent place to gain experience due to the variety of patients. The complexity of their conditions offers a great challenge to all members of staff, and I think they find it very interesting and enjoyable to work here. 